\title{
Pressure stimulated currents in rocks and their correlation with mechanical properties
}

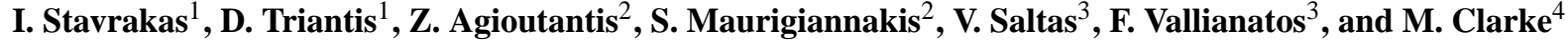 \\ ${ }^{1}$ Department of Electronics, Technological Educational Institution of Athens, Greece \\ ${ }^{2}$ Department of Mineral Resources Engineering, Technical University of Crete, Greece \\ ${ }^{3}$ Department of Natural Resources and Environment, Technological Educational Institution of Crete, Greece \\ ${ }^{4}$ Department of Information Systems and Computing, Brunel University, UK
}

Received: 30 June 2004 - Revised: 5 October 2004 - Accepted: 6 October 2004 - Published: 8 October 2004

Part of Special Issue "Precursory phenomena, seismic hazard evaluation and seismo-tectonic electromagnetic effects"

\begin{abstract}
The spontaneous electrification of marble samples was studied while they were subjected to uniaxial stress. The Pressure Stimulated Current (PSC) technique was applied to measure the charge released from compressed Dionysos marble samples, while they were subjected to cyclic loading. The experimental results demonstrate that, in the linear elastic region of the sample, no PSC is recorded, while beyond the stress limit ( $\mathrm{s}>0.60$ ), observable variations appear, which increase considerably in the vicinity of sample failure, reaching a maximum value just before the failure. The emitted current is reduced on each loading cycle and it has a reciprocal dependence to the normalized Young modulus. The MCD model, applied out of the vicinity of sample failure explains successfully the above findings. The existence of a "memory-like" behavior of the sample, could justify the weakness or absence of electrical earthquake precursors, during an aftershock sequence.
\end{abstract}

\section{Introduction}

The application of uniaxial compression in rock samples is accompanied by the generation of weak electric currents, which are known as Pressure Stimulated Currents (PSC). In order to understand the mechanisms that produce these transient electric signals during the loading process prior to and concurrently with rupture, many fracture laboratory experiments have been conducted using various minerals and rocks under both dry and saturated conditions (e.g. Nitsan, 1977; Ogawa et al., 1985; Brady and Rowell, 1986; Cress et al., 1987; Yamada et al., 1989; Enomoto and Hashimoto, 1990; Hadjicontis and Mavromatou, 1994, 1995; O'Keefe and Thiel, 1995; Freund, 2000; Takeuchi and Nagahama, 2001). A deep understanding of the involved mechanisms is

Correspondence to: D. Triantis

(triantis@ee.teiath.gr) essential for a further microscopic correlation with observed electric earthquake precursors (Vallianatos et al., 2004).

Among the different proposed electrification mechanisms, a particularly promising effect that is ubiquitous during brittle rock fracture is the motion of charged edge dislocations (moving charged dislocations, MCD) during crack formation and propagation at the terminal phase of the deformation cycle (Vallianatos and Tzanis, 1998, 1999a, 1999b; Tzanis and Vallianatos, 2002). In previous work (Stavrakas et al., 2003; Anastasiadis et al., 2004) PSC recordings have been reported during mechanical stress application on rock samples with various stress modes. The experimental results are satisfactorily supported by the MCD model. According to this model, when the material is uniaxialy compressed with time varying stress $S$, the Pressure Stimulated Current I can be estimated by the following relationship:

$I=\gamma \cdot\left(\frac{d S}{d t}\right)$

where $d S / d t$ is the stress rate and $\gamma$ is a scaling factor, which has a reciprocal dependence on the Young's modulus of the material. In the elastic region the stress $S$ on the material is related to the deformation $\varepsilon$ (i.e. strain) according to the following linear law:

$S=Y_{o} \cdot \varepsilon$

where $Y_{o}$ is the Young modulus of the undamaged material. When the stress takes values beyond the (linear) elastic range then microcracks occur, the sample is plastically deformed and the strain $\varepsilon$ is greater than the value given by Eq. (2). Accordingly (Turcotte et al., 2003):

$S=Y_{e f f} \cdot \varepsilon$

where $Y_{\text {eff }}$ is the effective Young's modulus which is no longer constant. In the plastic region the Young's modulus becomes progressively smaller while stress increases. 


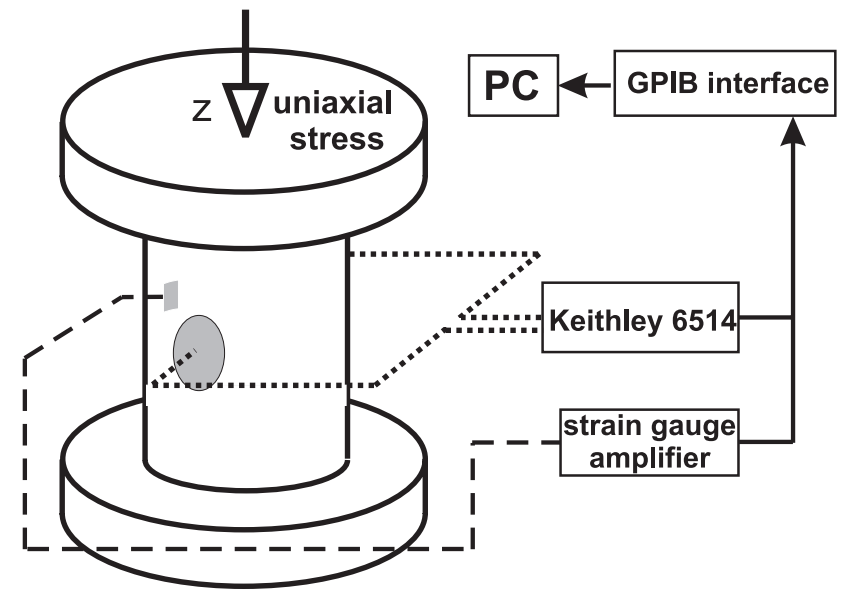

Fig. 1. Experimental setup for PSC, stress and strain measurements.

Regarding the scaling factor $\gamma$, the following proportionality describes its behavior as a function of the Young's modulus:

$\gamma \propto \frac{1}{Y_{e f f}} \propto \frac{1}{Y_{e f f} / Y_{o}} \propto \frac{1}{y}$

where $y$ stands for the Young modulus normalized to $Y_{O}$ value.

Using $y$, Eq. (1) can be expressed as follows:

$I \propto \frac{1}{y} \cdot\left(\frac{d S}{d t}\right)$

Whether the above MCD model, as well as, other possible electrification mechanisms can scale up to earthquake-size volumes and produce observable electrical earthquake precursors, is still under investigation. However, the mechanical conditions of the deforming material and its loading history could be associated successfully with the macroscopic field observations of weakness or absence of electric precursors, during an earthquake sequence.

In the present work, Dionysos marble samples have been subjected to cyclic loading - unloading and PSCs have been recorded at a direction perpendicular to the applied load. The experimental results are discussed in the context of the MCD model and in relation to a damage variable that is related to the variation of the Young's modulus of the samples under investigation.

\section{Experimental setup}

Figure 1 presents the complete experimental setup including both the mechanical parts that apply uniaxial compression and the electronic parts that perform the PSC measurements. The stress is applied by a loading unit (model MTS815 ) capable of a maximum force of $\pm 1600 \mathrm{kN}$ and a maximum displacement of $\pm 50 \mathrm{~mm}$. An intergrated electronic microconsole (model MTS-453.20), equipped with a load and displacement controller as well as a function generator

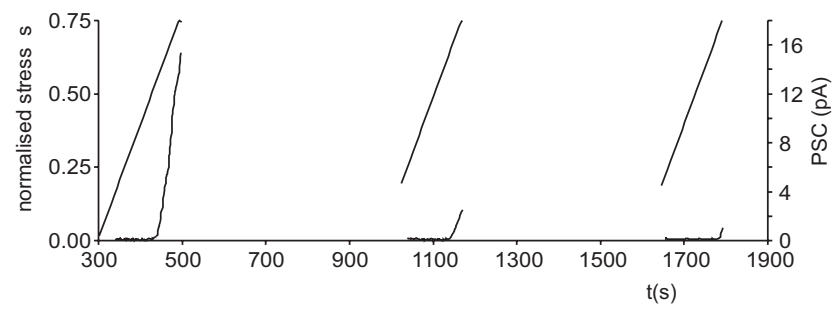

Fig. 2. PSCs recordings during the three sequential loading cycles in the range of the normalized stress, $0<s<0.75$.

unit, is used to provide closed loop control of the servohydraylic system. A detailed description of the loading apparatus is given elsewhere (Kleftakis et al., 2000). Cylindrical samples (51 $\mathrm{mm}$ in diameter and $106.4 \mathrm{~mm}$ in height) were placed between two thin teflon plates in order to provide electrical insulation. A pair of electrodes was attached to each marble sample, using conductive paint. The electrodes were placed in a direction perpendicular to the axis of the applied stress. For conducting electrical measurements a sensitive programmable electrometer (Keithley 6514) with a current range from $0.1 \mathrm{fA}$ to $20 \mathrm{~mA}$, has been used measuring at a direction perpendicular to the applied stress direction. The experiment has been conducted in a Faraday shield to prevent measurements from being affected by electric noise.

The geomaterials used to perform the experiments were Pentelicon marbles (Dionysos) samples collected from Penteli Mt., Attica. Their chemical composition is $98 \%$ calcite and $0.2 \%$ of quartz and other minerals such as muscovite, sericite and chlorite (Kleftakis et al., 2000). Its density is $2.7 \mathrm{Mg} / \mathrm{m}^{3}$, its porosity is $0.371 \%$ and its absorption coefficient by weight is $0.11 \%$.

\section{Experimental results and discussion}

Preliminary uniaxial compression tests were carried out in order to determine the mechanical behavior of the samples under investigation. The results demonstrate that all the samples exhibit a typical stress - strain variation with the elastic behavior reaching approximately $60 \%$ of the failure stress of samples (102 MPa) (Stavrakas et al., 2004).

Afterwards, the samples were subjected to three loading - unloading cycles with a constant stress rate. The absolute value of the stress rate, during both loading and unloading cycles was $241 \mathrm{kPa} / \mathrm{s}$. During the first two loadings, the normalized stress varied in the limits of $0<s<0.75$ and $0.2<s<0.75$, while in the last loading the stress gradually reached $s=1$, where the sample failed. Immediately after completing the first and second loading cycle, the stress remained at a non zero value $(s=0.2)$ for a significant period of time so the PSC could relax to a minimum value.

The variation of the emitted PSCs in comparison with the normalized stress variation during the three loadings is depicted in Fig. 2. The PSC emitted during the first loading cycle (PSC-1) is significantly higher than the corresponding 


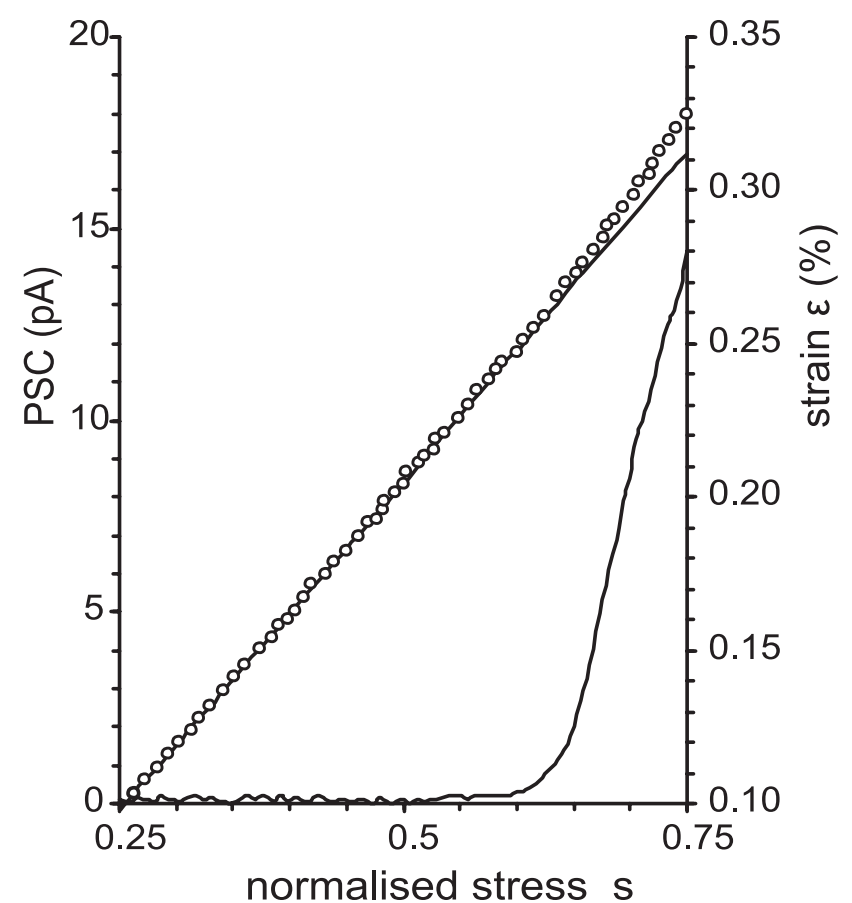

Fig. 3. PSC-1 recordings as a function of normalized stress, s. The stress - strain variation for $s$ values up to 0.75 is also shown. The dotted line represents the extension of the linear (elastic) region.

PSCs released during the second and third loadings (PSC-2 and PSC-3). The fact that the amount of the total charge, which is released during each loading cycle, is reduced with the time evolution of the experiment, may be explained in terms of the "work hardening" effect. According to this process, repetitive stress - strain tests may affect the elastic properties of the marble samples. When stress is applied to a "pristine" marble sample and drives the material into the plastic deformation range, as it happens in this case, dislocations move and multiply and microcracks occur. On applying stress for the second time, many of the existing dislocations are replaced by neighboring partial dislocations as this process results in a reduction of the elastic energy of the sample (Feltham, 1966). This process does not necessarily result in an increase of the released charge, which participates in the PSC and consequently a weaker current is generated.

In order to compare the mechanical behavior of the sample during the uniaxial compression and the corresponding PSC emission, the variation of both, strain and PSC-1, with respect to the normalized stress $s$ of the first loading have been plotted (Fig. 3). As seen in Fig. 3, the linear part of the stress - strain curve extends up to $s=0.60$. For $s>0.60$, the curve deviates from linearity and the sample enters gradually to the plastic deformation region. Before significant deformation takes place, the stress is decreased with the same rate to the value of $s=0.20$.

For stress $s<0.60$, the PSC does not exhibit significant variations and varies close to the electrometer's noise background. The latter is consistent with Eq. (1), because the

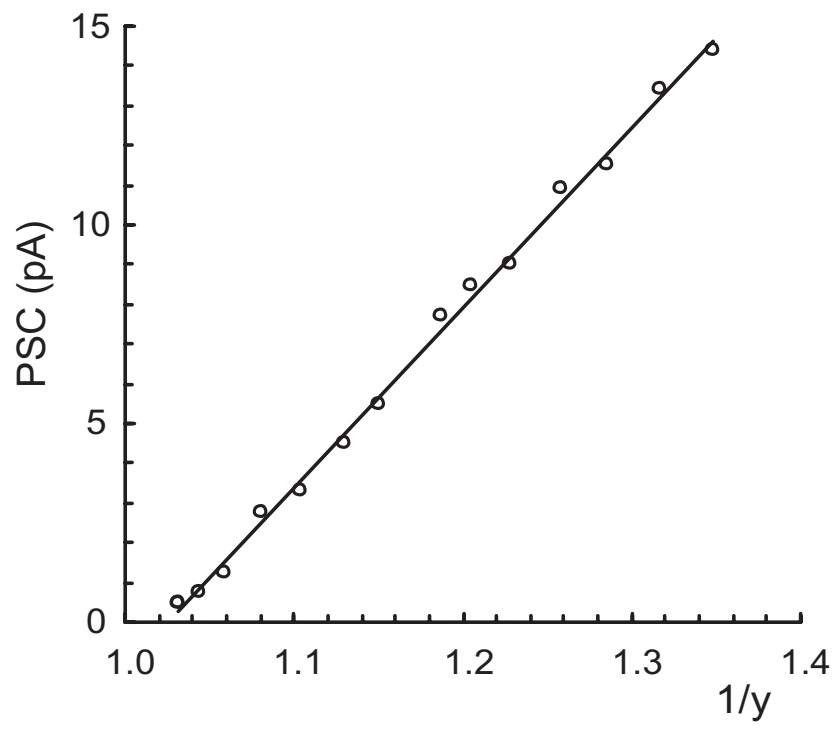

Fig. 4. Variation of the emitted PSC-1 versus $1 / y$ (Eq. 1), in the range $0.60<s<0.75$.

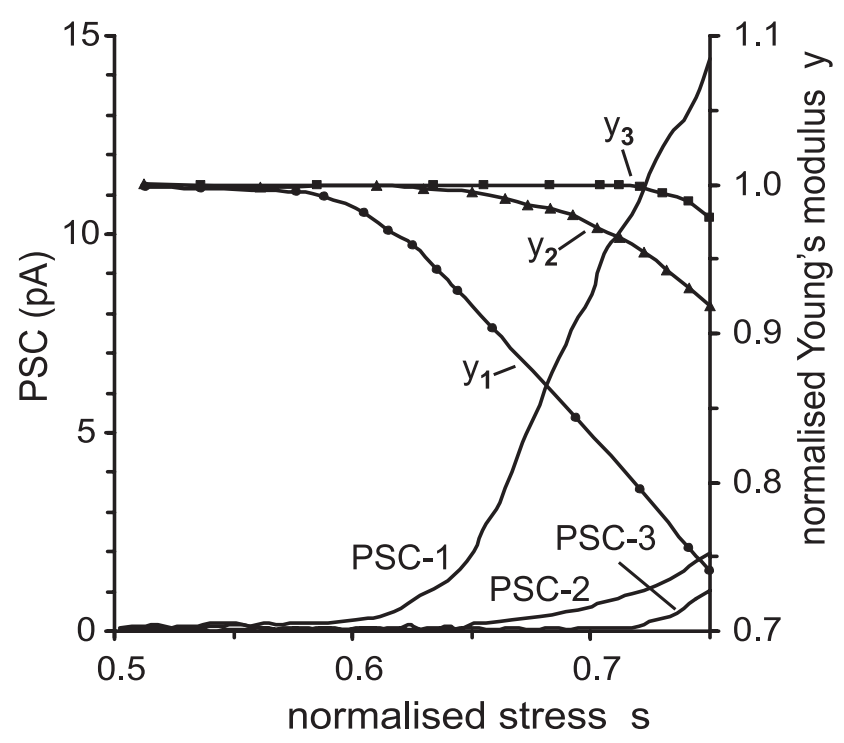

Fig. 5. Variations of the recorded PSCs (solid lines) and the corresponding Young modulus $y$ with respect to the normalized stress, during the three loading processes.

scaling factor $\gamma$ is constant, since the Young modulus in this elastic region is also constant.

Observable variations at the recorded PSC appear beyond the stress limit $(s=0.60)$, where $Y=Y_{\text {eff }}<Y_{o}$. The variation of the emitted PSC-1 versus the inverse of the normalized Young modulus, $y$, for the stress range $0.6<s<0.75$ has been plotted in Fig. 4. The observed linear relation between PSC and $1 / y$ is in complete agreement with Eq. (3), which predicts this proportionality, taking into account that the stress rate remains constant.

Figure 5 illustrates in detail the differences at the initiation of the three recorded PSCs during the loading cycles. 


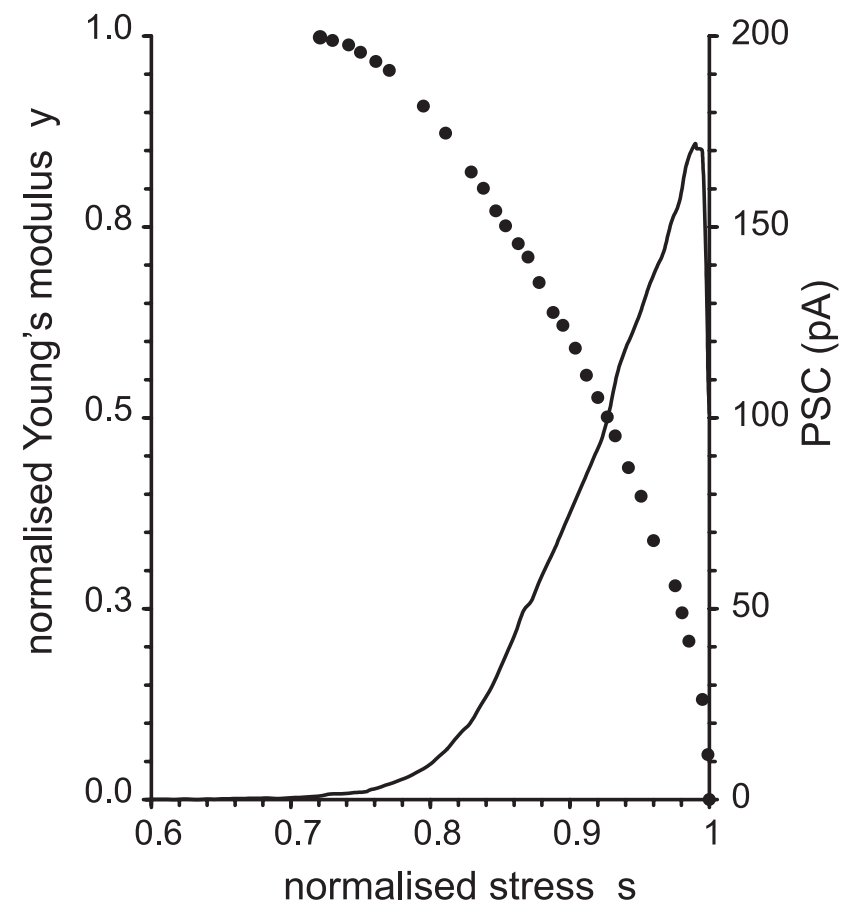

Fig. 6. Variations of the PSC emitted during the last loading cycle (PSC-3) and the corresponding normalized Young's modulus, with respect to the normalized uniaxial stress $s$.

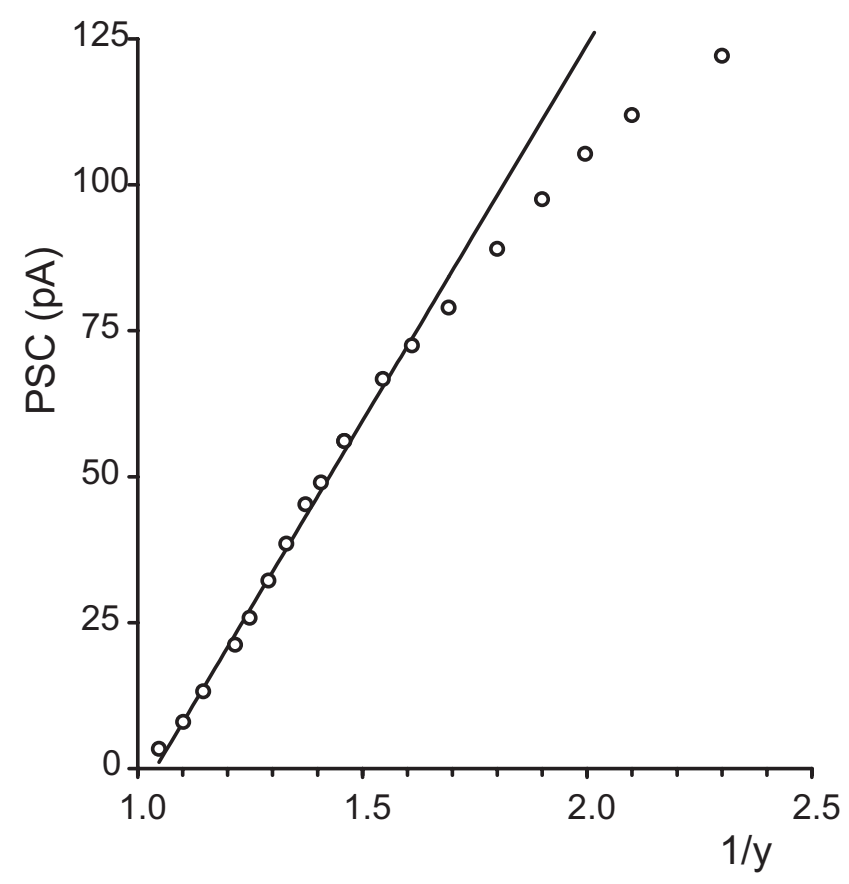

Fig. 7. Correlation of the emitted PSC with respect to the $1 / y$ factor of the last loading cycle.

Additionally, the reduced Young's modulus $y=Y_{\text {eff }} / Y_{o}$, as calculated by the fitting of the corresponding stress - strain curves, is plotted versus the normalized uniaxial compressive stress $s$. According to Fig. 5, the initiation of the recorded PSCs is observed when the normalized Young's modulus $y$

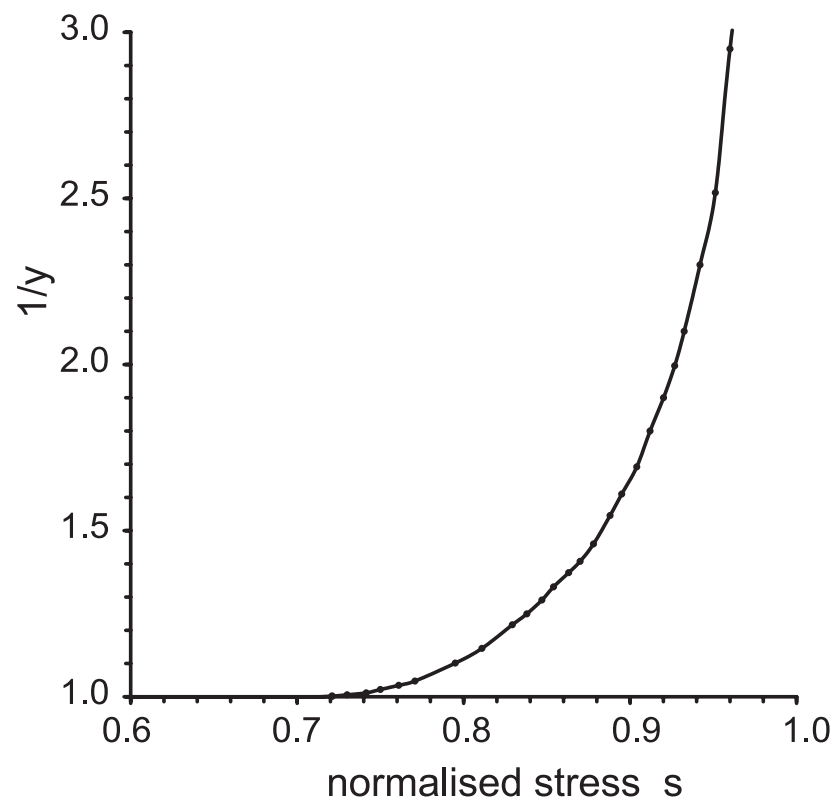

Fig. 8. Correlation of the $1 / y$ factor with respect to the normalized stress values of the last loading cycle.

exhibits values lower than 1 . For a given value of the normalized uniaxial stress $s$ that corresponds to $y<1$ PSCs with values $I_{1}>I_{2}>I_{3}$ are recorded during the three sequential loading processes. This observation is consistent with Eq. (3), taking into account that for the corresponding y values of the measured PSCs, the relation $y_{1}<y_{2}<y_{3}$ is valid during the three loading processes.

The PSC emitted during the last loading cycle (PSC-3) and the corresponding normalized Young's modulus y with respect to the normalized uniaxial stress $s$, are shown in Fig. 6. At normalized stress values very close to unity (i.e. just before failure), a sharp reduction of PSC was recorded. Such a behaviour of the PSC just before the failure has been observed in similar experiments, in a previous work (Stavrakas et al., 2003).

Figure 7 demonstrates the variation of the emitted PSC versus the $1 / y$ factor at the last loading cycle, which results to sample failure. A linear relation is observed for the $1 / \mathrm{y}$ values in the range $1<1 / y<1.65$. Beyond this limit, the curve deviates from linearity. A correlation of the $1 / y$ factor with respect to the normalized stress values is shown separately in Fig. 8. According to this figure, for $1 / y<1.65$ the normalized stress value is less than 0.9. As a consequence, Eq. (3) is valid only below this upper stress limit. For $s>0.9$, i.e. just before the failure, the emitted current has a non - linear dependence with the $1 / y$ factor.

The above observations imply that Eq. (3), is not applicable near the vicinity of sample failure, since it presupposes that the number of the dislocations remain constant during the stress application. An additional term should be included, taking into account the massive dislocation production just before failure, which contributes further to the build up of pressure stimulated currents. 


\section{Conclusions}

In the present work, the spontaneous electrification of marble samples is studied by means of Pressure Stimulated Current (PSC) technique and the findings are discussed in relation to the mechanical properties of the samples, in the context of the moving charged dislocation (MCD) model. Dionysos marble samples were subjected to a cyclic loading with a constant absolute stress rate and PSCs were recorded. The evaluation of the experimental results suggests the followings.

1. No PSC is recorded when the sample is compressed in the linear elastic region.

2. Observable variations at the recorded PSCs appear beyond the linear eleasticity limit of the sample $(s>0.60)$, while in the vicinity of sample failure PSCs increase considerably, reaching a maximum value just before failure.

3. The emitted current during sequential loading cycles has a reciprocal dependence to the normalized Young modulus for stress values out of the vicinity of sample failure.

4. The amount of the released charge is reduced on each loading cycle, due to the work hardening effect.

The above "memory effect" of the rock samples under sequential loading cycles, could justify the weakness or absence of electric eartquake precursors during an aftershock sequence, subject under investigation and debate in seismoelectromagnetic research (see Geller, 1996).

Edited by: P. F. Biagi

Reviewed by: A. Tzanis and another referee

\section{References}

Anastasiadis, C., Triantis, D., Stavrakas, I., and Vallianatos, F.: Pressure Stimulated Currents (PSC) in marble samples, Annals of Geophysics, accepted, 2004.

Brady, B. T. and Rowell G. A.: Laboratory investigation of the electrodynamics of rock fracture, Nature, 321, 488-492, 1986.

Cress, G. O., Brady B. T., and Rowell G. A.: Sources of electromagnetic radiation from fracture of rock samples in laboratory, Geophys. Res. Lett., 14, 331-334, 1987.

Enomoto, J. and Hashimoto H.: Emission of charged particles from indentation fracture of rocks, Nature, 346, 641-643, 1990.

Feltham, P.: Deformation and Strength of Materials, Butterworths, 1966.

Freund, F.: Time-resolved study of charge generation and propagation in igneous rocks, J. Geophys. Res., B105, 11 001-11 019, 2000.

Geller, R. J.: VAN: A critical enaluation, in: A Critical Review of VAN, edited by: Sir J. Kighthill, World Scientific, Singapore, 155-238, 1996.
Hadjicontis, V. and Mavromatou, C.: Transient electric signals prior to rock failure under uniaxial compression, Geophys. Res. Lett., 21, 1687-1690, 1994.

Hadjicontis, V. and Mavromatou, C.: Electric signals recorded during uniaxial compression of rock samples: Their possible correlation with preseismic electric signals, Acta Geophysica Polonica, Vol. XLIII, No.1., 1995.

Kleftakis, S., Agioutantis, Z., and Stiakakis, C.: Numerical simulation of the uniaxial compression test including the specimenplaten interaction, Computational methods for shell and spatial structures, Proceedings CD-ROM, IASS-IACM, 2000.

Nitsan, U.: Electromagnetic emission accompanying fracture of quartz-hearing rocks, Geophys. Res. Lett., 4, 533, 1977.

O'Keefe, S. G. and Thiel, D. V.: A mechanism for the production of electromagnetic radiation during fracture of Brittle materials, Physics of the Earth and Planetary Interiors, 89. p., 127-135, 1995.

Ogawa, T., Oike, K., and Miura, T.: Electromagnetic radiations from rocks, J. Geophys. Res., 90, D4, 6245-6249, 1985.

Stavrakas, I., Anastasiadis, C., Triantis, D., and Vallianatos, F.: Piezo Stimulated currents in marble samples: Precursory and concurrent with failure signals, Natural Hazards and Earth System Sciences, 3, 243-247, 2003,

SRef-ID: 1684-9981/nhess/2003-3-243.

Stavrakas, I., Triantis, D., Agioutantis, Z., Maurigiannakis, S., Saltas, V., and Vallianatos, F.: Pressure Stimulated Currents along with mechanical properties in rocks, Proceedings, Advances in Mineral Resources Management and Environmental Geotechnology, Hania, June 7-9, 2004.

Takeuchi, A. and Nagahama, H.: Voltage changes induced by stickslip of granites, Geophys. Res. Lett., 28, 3365-3367, 2001.

Turcotte, D. L., Newman, W. I., and Shcherbakov, R.: Micro and macroscopic models of rock fracture, Geophys. J. Int., 152, 718728, 2003.

Tzanis, A. and Vallianatos, F.: A physical model of electrical earthquake precursors due to crack propagation and the motion of charged edge dislocations, in: Seismo Electromagnetics: Lithosphere-Atmosphere-Ionosphere Coupling, edited by: Hayakawa, M. and Molchanov, O. A., TERRAPUB, Tokyo, 117130, 2002.

Vallianatos, F. and Tzanis A.: Electric current generation associated with the deformation rate of a solid: Preseismic and coseismic signals, Phys. Chem. Earth, 23, 933-938, 1998.

Vallianatos, F. and Tzanis, A.: A model for the generation of precursory electric and magnetic fields associated with the deformation rate of the earthquake focus, in: Atmospheric and Ionospheric electromagnetic phenomena associated with Earthquakes, edited by: Hayakawa, M., Terra Scientific Publishing Co., Tokyo, 287305, 1999a.

Vallianatos, F. and Tzanis A.: On possible scaling laws between Electric Earthquake Precursors (EEP) and Earthquake Magnitude, Geophys. Res. Lett., 26 (13), 2013-2016, 1999b.

Vallianatos, F., Triantis, D., Tzanis, A., Anastasiadis, C., Stavrakas, I.: Electric Earthquake Precursors: From Laboratory Results to Field Observations, Phys. Chem. Earth., 29, 339-351, 2004.

Yamada, I., Masuda, K., and Mizutani, H.: Electromagnetic and acoustic emission associated with rock fracture, Phys. Earth Planet Inter., 57, 157-168, 1989. 